\title{
Optimization of Hybrid Satellite Constellations using Multiple Layers and Mixed Circular-Elliptical Orbits
}

\author{
Serena Chan ${ }^{*}$, Ayanna T. Samuels ${ }^{\dagger}$, Nirav B. Shah ${ }^{\ddagger}$, Jennifer E. Underwood ${ }^{\S}$, and Olivier L. de Weck \\ Massachusetts Institute of Technology, Cambridge, MA 02139, USA
}

\begin{abstract}
Previous research in satellite constellation designs has focused on Low Earth Orbit (LEO) communication systems that service a global market of uniform demand. This paper addresses the challenge of designing a hybrid satellite system constellation to meet the emerging satellite broadband market while enabling a phased satellite deployment strategy. The broadband market model developed in the paper highlights the highly non-uniform distribution of traffic that future satellite systems must handle. The proposed solution employs a LEO backbone satellite constellation to capture a fraction of the total market demand with additional elliptical satellite constellations to service areas of high and growing demand. By opening up the tradespace to multiple orbital altitudes (Low Earth Orbit, Medium Earth Orbit, and Geosynchronous Orbit) and both circular and elliptical orbits, this paper will search for a lower cost solution to the broadband communication constellation design problem. Lastly, this paper will introduce a novel visualization of the tradespace using convex hulls and conditional Pareto fronts, which enable instant recognition of the impact of design choices on the final design.
\end{abstract}

\section{Introduction}

Satellite constellation design is a complex process that requires evaluation of many issues, including analysis of Norbit characteristics. The fundamental rationale for deploying multiple satellites is to provide greater Earth coverage. A single satellite cannot provide as many observations nor as frequent communications as multiple satellites can. Unfortunately, there are neither absolute rules nor a standard policy for designing a constellation. The complexity of the problem demands that in choosing a preliminary design, alternate designs must be carefully assessed and reasons for the final choices must be documented.

A discussion of the key issues and orbit characteristics necessary for the constellation design process is provided by Wertz and Larson (1999). ${ }^{1}$ Use of circular polar orbit constellations have been shown by Rider (1985) to be efficient for providing highly redundant Earth coverage. ${ }^{2}$ Adams and Rider (1987) have extended that work to analyze circular polar constellations providing single or multiple coverage above a specified latitude. ${ }^{3}$ Lang and Adams (1998) and Turner (2002) highlight utilizing Walker patterns as another candidate constellation for providing continuous Earth coverage. ${ }^{4,5}$ Numerous circular polar and Walker constellations are examined by de Weck and Chang (2002) in their development of a methodology for examining the architecture trade space for LEO personal communication systems. ${ }^{6}$

\section{A. Problem Motivation}

There are two important underlying assumptions common to previous research in satellite constellation design. First, all satellites within a constellation must follow circular orbits and maintain the same altitude. Alternate designs with varying satellite altitudes should be considered. Second, the market demand for satellite communication services is uniformly distributed across the globe. This assumption poorly models the amount of traffic seen by the satellites given the non-uniform distribution of the population on the Earth and of the potential customers' desire for or ability to access satellite communication services. This paper relaxes these two common assumptions and

\footnotetext{
${ }^{*}$ Research Assistant, Laboratory for Information and Decision Systems, AIAA Student Member.

${ }^{\dagger}$ Research Assistant, Space Systems Laboratory, AIAA Student Member.

${ }^{\ddagger}$ Research Assistant, Lean Aerospace Initiative, AIAA Student Member.

$\S$ Draper Laboratory Fellow, Charles Stark Draper Laboratory, AIAA Student Member.

"Assistant Professor of Aeronautics \& Astronautics and Engineering Systems, AIAA Member.
} 
explores the design of a hybrid satellite system constellation to meet the emerging satellite broadband market. In other words, an attempt is made to judiciously match system capacity with demand.

The hybrid satellite system consists of a backbone of LEO satellites serving nominal levels of demand at all points on the globe and a fleet of elliptical satellites serving concentrated areas of high demand. In order to capture this non-uniform demand, the satellite broadband market must first be identified. The satellite broadband market data are collected from Kashitani (2002). ${ }^{8}$ The market demand is determined by using the Gross National Product (GNP) adjusted by Purchasing Power Parity (PPP) (i.e., converting GNP to international dollars), as shown in the GNP PPP Map in Fig.1, and using the worldwide population distribution, as shown in the Population Map of Fig. 1. The international dollar is equivalent to the United States dollar. The GNP PPP Map and the Population Map of Fig. 1 are then combined to create the market demand for satellite broadband services. The derivation of the market demand is described in Section III and illustrated in Fig. 3.

\section{GNP PPP Map}
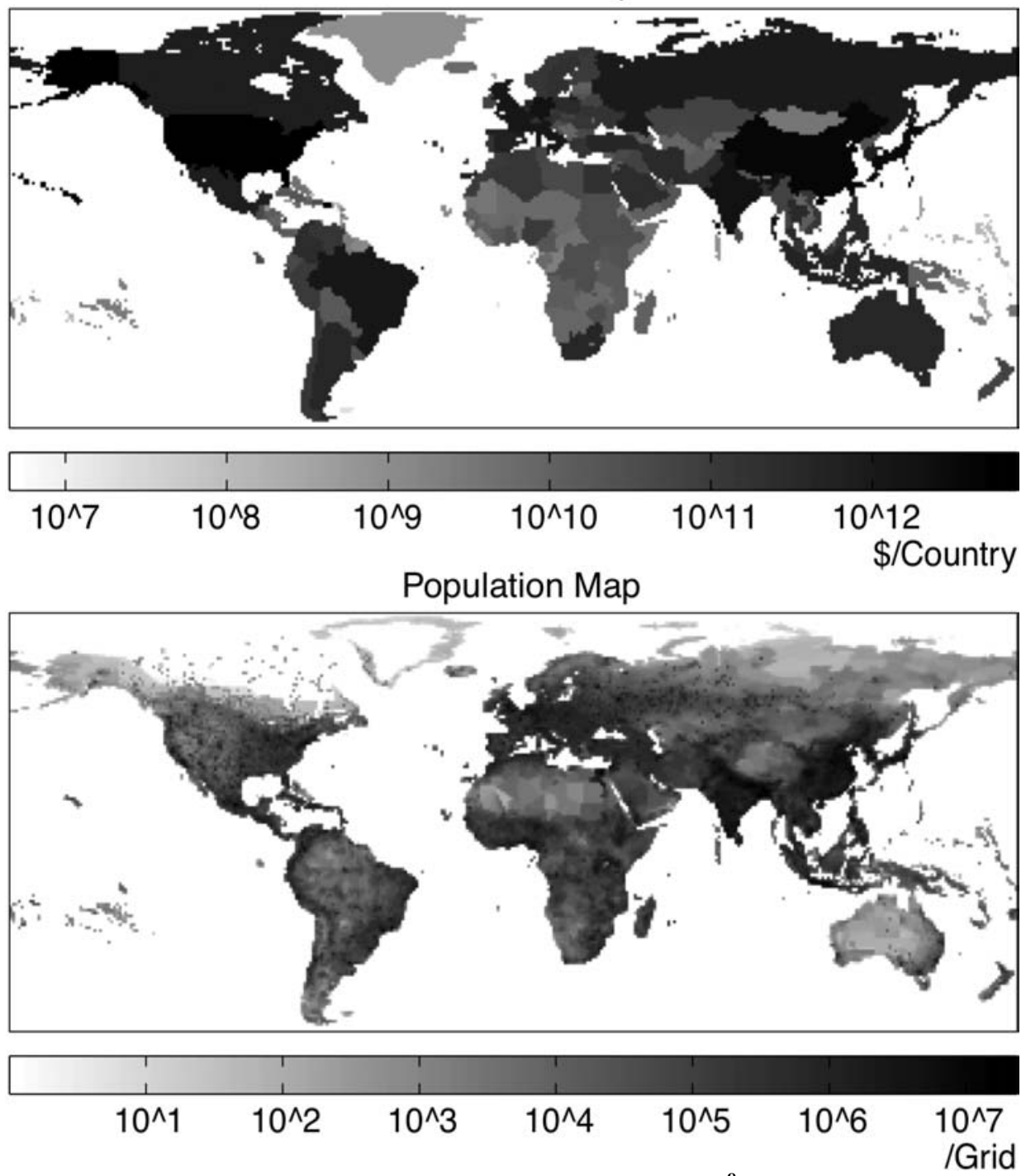

Figure 1. Global Distribution Maps. ${ }^{8}$

Figure 2 shows a bar graph of the longitudinal demand structure of the hybrid system summed across all latitudes. Above a certain minimum threshold, shown notionally as 200 users in Figure 2, the demand distribution is highly non-uniform and localized into several peaks. To capture such a non-uniform demand, a two-phase strategy is suggested. The first phase consists of a backbone constellation that provides global coverage with capacity up to the threshold level. The second phase consists of an elliptical constellation arranged so that apogee occurs above areas of high demand, thereby providing additional capacity only where it is demanded. The elliptical constellation need 
not be deployed all at once. Rather, individual planes that focus on particular demand 'hot-spots' can be deployed as the market develops and regulatory approvals are obtained. This can be a cost-efficient strategy that prevents underutilization of the constellation.

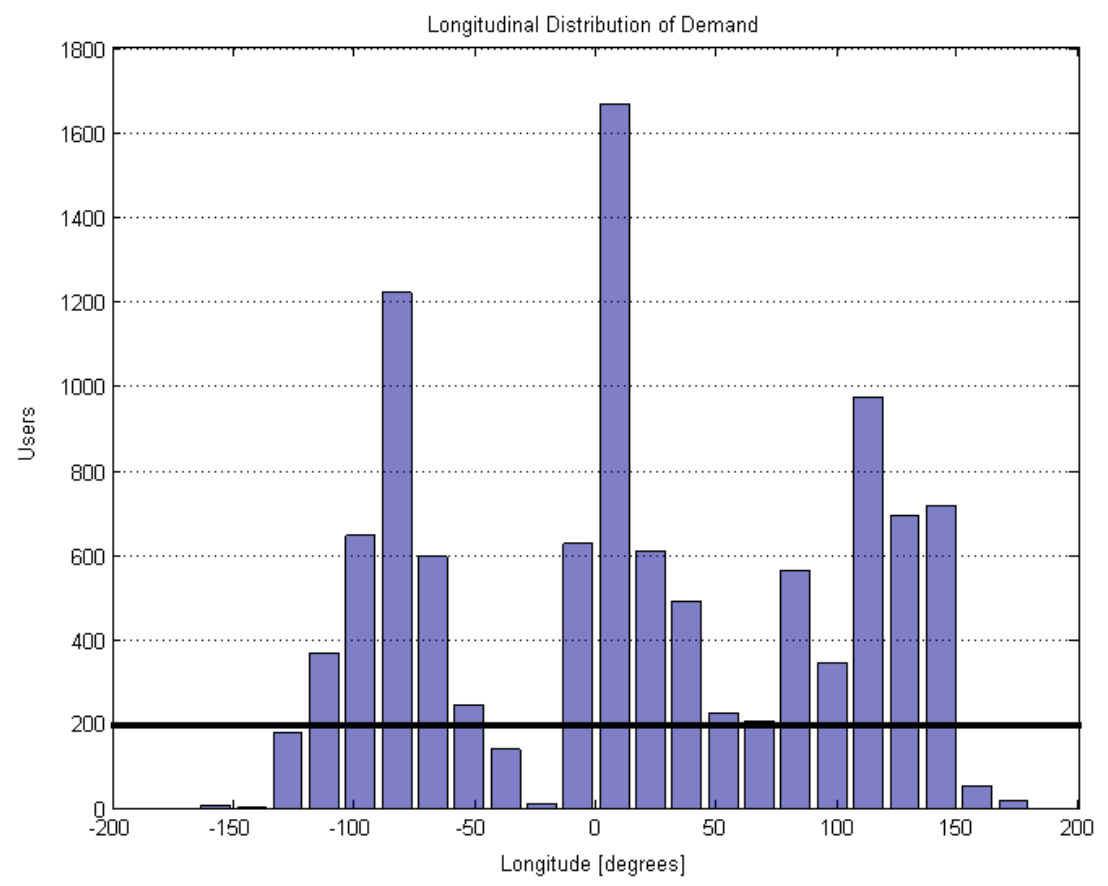

Figure 2. Longitudinal Distribution of Demand Showing Number of Users vs. Longitude (Summed Across All Latitudes)

In the following sections, the problem is defined and a full factorial search of the elliptical system tradespace is conducted to determine the final choice of a low-cost hybrid satellite constellation with enough capacity to meet the needs of the emerging satellite broadband market. A Pareto front of the set of optimal hybrid satellite system designs that solve the formulated optimization problem is given.

\section{Problem Definition and Approach}

The purpose of this paper is to present the design and optimization of a hybrid satellite system constellation. The systems architecture problem is rationalized and quantified by selecting appropriate design variables, objective functions, and constraints. Methods and tools in multidisciplinary system design optimization are implemented and discussed. Interpretation and analysis of optimization results, including a discussion of sensitivity studies and an exploration of performance and cost tradeoffs are also provided.

The optimization of a hybrid satellite system can be decomposed into two separate optimization problems. In one problem, the LEO backbone is optimized to deliver the threshold capacity at minimum cost. The second problem involves optimizing the elliptical portion. Existing design methods for LEO constellations (e.g. de Weck and Chang $(2002)^{6}$ ) can be used to solve the first problem. Such techniques, however, are not applicable to the second problem because of the relaxation of the assumptions mentioned in the previous section. Therefore, this paper focuses on the problem of architecting the elliptical portion of the hybrid system.

The architecture problem for the elliptical system can be stated as follows:

"How should the satellite constellation orbit, period, eccentricity, number of planes, satellite transmit power, and antenna diameter be sized for a given elevation angle, market demand distribution, channel bandwidth, and satellite lifetime, while minimizing cost and maximizing system throughput?"

\section{A. Problem Formulation}

The primary objective of the optimization problem is to minimize the lifecycle cost (LCC) of the hybrid satellite constellation architecture. Given that the LEO backbone constellation is not optimized here, its cost is considered to 
be a fixed charge paid regardless of the design of the elliptical system. The elliptical system is constrained to meet at least $90 \%$ of market demand. Note that when referring to market demand in the context of the elliptical system, only demand above the threshold satisfied by the backbone is included.

A secondary objective of maximizing the unused capacity of the system is included to allow study of the tradeoff between cost and the capacity available for future expansion. Defining the second objective requires some care. It is not simply the total system capacity less the number of users (channels) served. There are design solutions that can place satellites in orbits covering areas where there is a lack of users, thus leading the simulation to conclude that there is a lot of "excess" capacity. This is clearly not the meaning of excess capacity that is useful for this optimization problem. Therefore, an alternative definition is required:

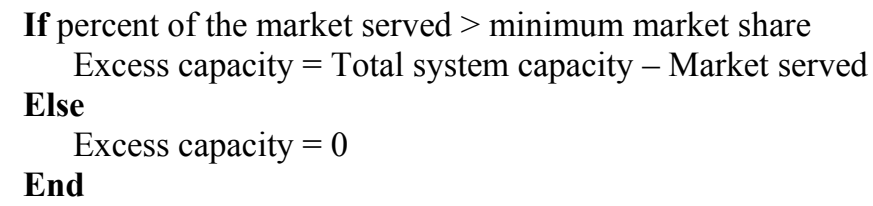

Because the market served varies over time, the second objective is defined as the average excess capacity (AEC).

These two objectives are mutually opposing. Maximizing the unused capacity of the satellite system will necessarily require either an increase in the number of satellites in the constellation or an increased capacity per satellite, both of which increase cost. The objective of minimizing the total lifecycle cost will attempt to drive down the number and cost of satellites, opposing the drive for excess capacity. Therefore, a single design will not be optimal for both objectives. Instead, a Pareto optimal set of designs is specified whose members optimize one objective for various fixed values of the other.

To make the design of the elliptical system amenable to computerized optimization, the tradespace of elliptical designs was parameterized using the design variables listed in Table 1. The orbital elements required for full specification of the orbit are calculated from the geographic demand distribution with the exception of the orbital period and eccentricity as these elements cannot be generated from the demand distribution. Hence, the orbital period and eccentricity are design variables. The number of planes specifies the number of ellipses used. The satellite transmitter power, the satellite antenna diameter, and the choice of a Multiple Access Scheme capture the design elements most critical for characterizing the communications capability of each satellite.

Table 1: Elliptical Constellation Design Vector.

\begin{tabular}{|l|l|c|}
\hline Symbol & Variable & Unit \\
\hline T & Orbital period & {$[$ Day $]$} \\
\hline E & Eccentricity & {$[-]$} \\
\hline NP & Number of planes & {$[-]$} \\
\hline Pt & Satellite transmitter power & {$[\mathrm{W}]$} \\
\hline DA & $\begin{array}{l}\text { Equivalent satellite antenna diameter } \\
\text { (Parabolic antenna) }\end{array}$ & {$[\mathrm{m}]$} \\
\hline MAS & Multiple Access Scheme (MF-TDMA or MF-CDMA) & {$[-]$} \\
\hline
\end{tabular}

\section{B. Problem Decomposition}

The problem of specifying the total lifecycle cost of the hybrid system can be decomposed into two parts: (1) design of the LEO backbone constellation and (2) optimization of the elliptical constellation. The total lifecycle cost of the entire system is the sum of the total lifecycle cost of the LEO backbone constellation and the total lifecycle cost of the elliptical constellation. Because the design of the LEO backbone constellation is based on validated optimization work conducted by de Weck and Chang $(2002)^{6}$, only slight modification of their simulation code is necessary to address the broadband market demand at nominal demand levels (e.g., number of subscribers, required data rate per user, average monthly usage, etc.). The next section discusses the development and validation of the simulation code necessary for optimizing an elliptical constellation to service the remaining market demand.

\footnotetext{
${ }^{\#}$ Equivalent satellite antenna diameter is used as a substitute for gain in the analysis.
} 


\section{Simulation Design}

\section{A. Simulation Assumptions}

Several assumptions were included in the hybrid simulation analysis. First, the LEO backbone satellites and the elliptical orbit satellites are constrained to be uniform (i.e., the same spacecraft design) but the two sets may not necessarily be the same; satellite lifetime is assumed to be 5 years. The inclination angles of the elliptical satellites are fixed at $63.4^{\circ}$. At this inclination, the perigee will not rotate, thus both apogee and perigee can be maintained over fixed latitudes. The elliptical orbits are also designed to use a repeating ground track to ensure specified longitudes are visited regularly.

It is assumed that the hybrid satellite system penetration ratio is such that the system satisfies the demand of one percent of the potential broadband satellite system market. Optical cross-links are used in order to force the communications bottleneck to occur in the ground-to-space segment, not in the space-to-space segment; they are assumed to provide a data rate of $3 \mathrm{Gbps}$. It is further assumed that the earth transceiver will have a high gain ${ }^{* *}$ characteristic of broadband users, who are assumed to demand a data rate of $1 \mathrm{Mbps}$ per user, sufficient to enable a diverse range of services including internet data, fax, and voice, and comparable with terrestrial consumer broadband data rates. The frequency spectrum used by the system is assumed to be the same as DirecTV's ${ }^{\dagger}$.

For computational efficiency, the resolution of the satellite broadband market demand map is reduced to square grids of $15^{\circ}$ latitude by $15^{\circ}$ longitude, as shown in Fig. 3. The demand distribution map is also normalized such that the sum of all elements equals one. The areas demanding the greatest satellite broadband services are located in the United States, Europe, and East Asia.
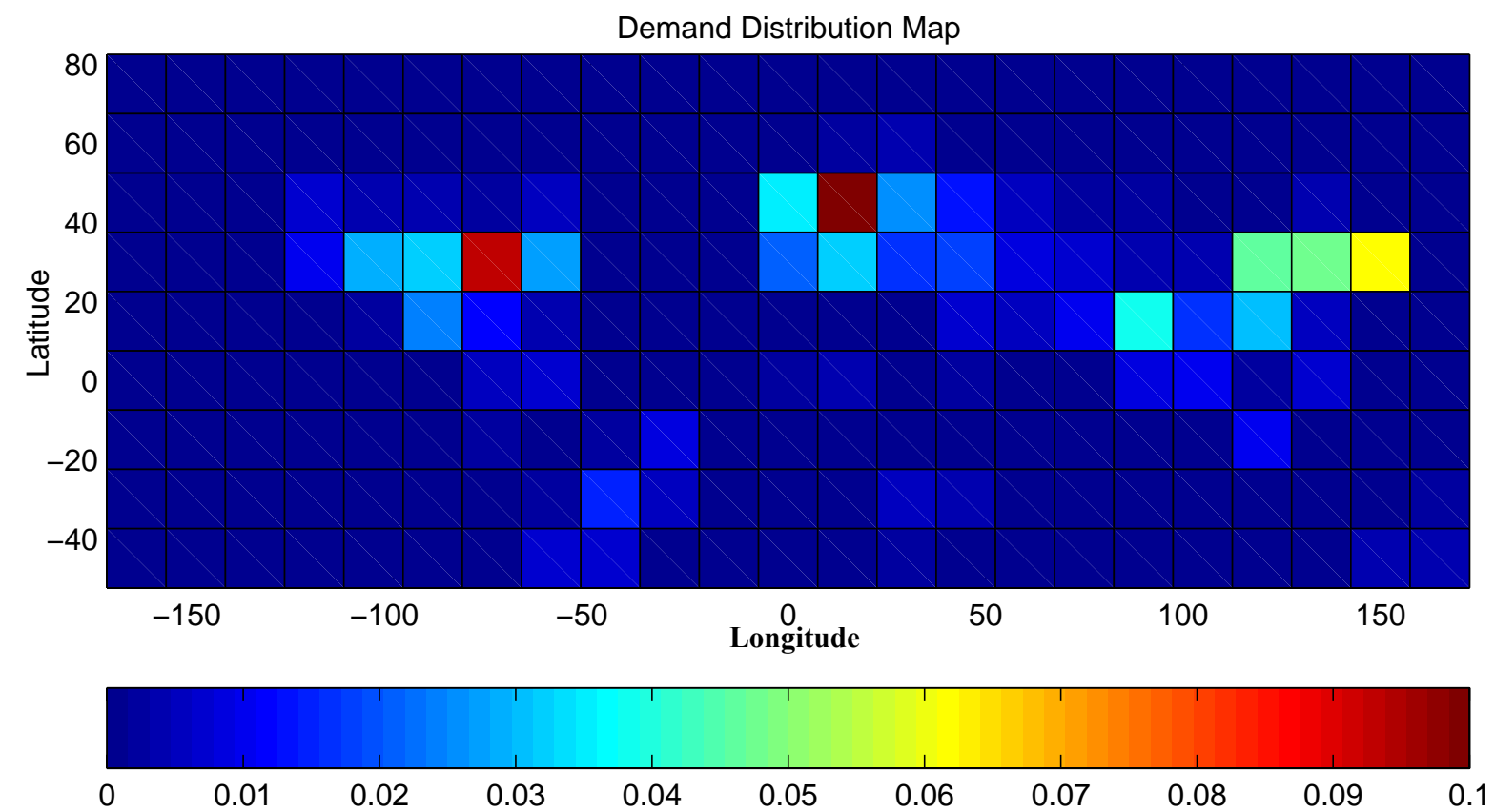

Figure 3. Market Demand Map Used in Simulation.

\section{B. Simulation Architecture}

The elliptical satellite constellation problem is decomposed into "black boxes," or modules, based on disciplinary tradition and degree of coupling of governing equations. A module in multidisciplinary system design optimization is defined as a finite group of tightly coupled mathematical relationships where some variables represent independent inputs while others are dependent outputs. ${ }^{9}$ Figure 4 illustrates the block diagram of the various simulation modules used for this paper.

\footnotetext{
** The gain used is $18 \mathrm{dBi}$.

${ }^{\dagger}$ DirecTV's bandwidth allocation is $17.3-17.8 \mathrm{GHz}$ on the uplink and $12.2-12.7 \mathrm{GHz}$ on the downlink.
} 


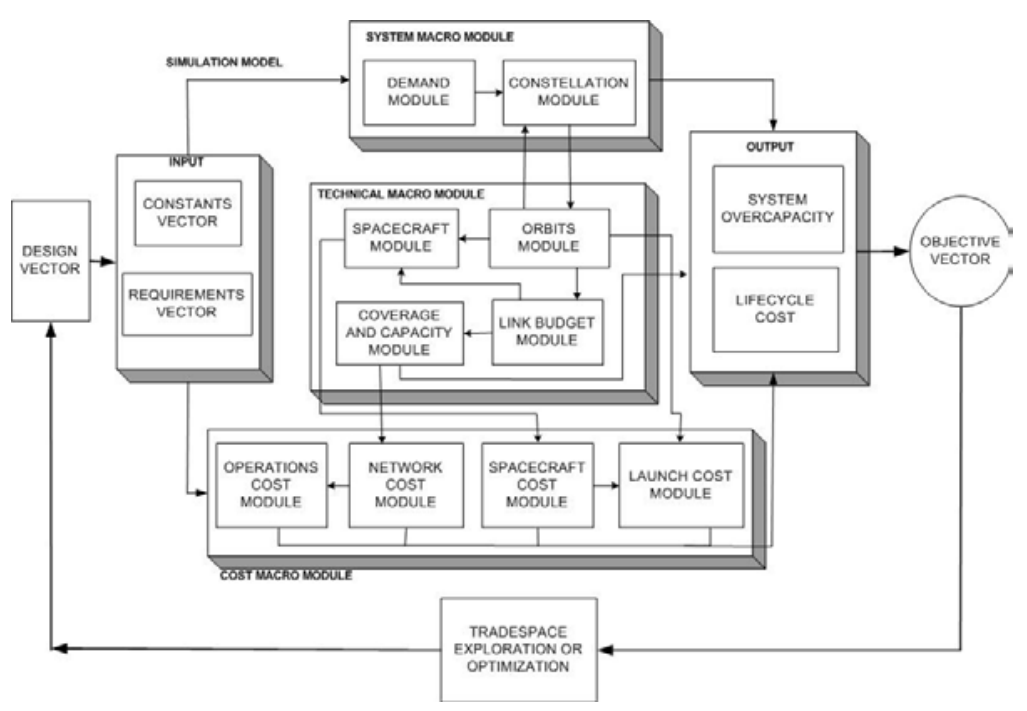

Figure 4. Elliptical Constellation Simulation Block Diagram.

To develop and organize interface information, an $N^{2}$ diagram is used. Each module within the simulation architecture is placed along the diagonal. Figure 5 provides a visual representation of the information flow through the simulation architecture. This technique is used to identify critical modules that have many inputs and outputs. Attempts have been made to minimize the number of feedback loops between modules. The fidelity of critical modules are then thoroughly tested and verified.

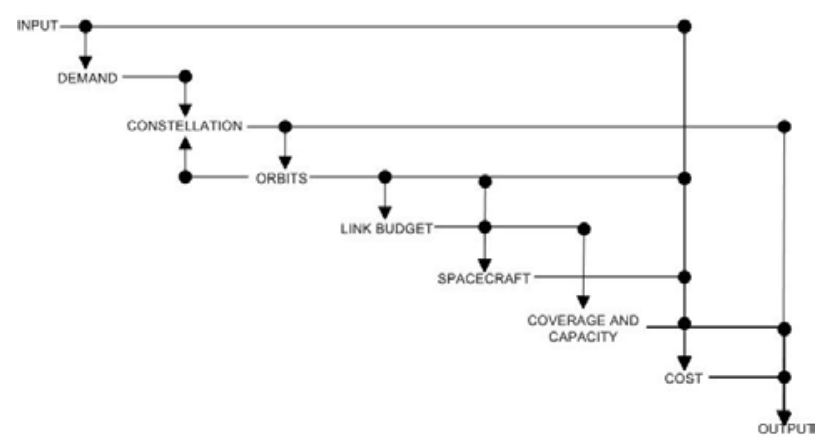

Figure 5. $N^{2}$ Diagram of Simulation Architecture.

A brief discussion of the important simulation modules, their validation, and their inherent assumptions, is provided in the following subsections.

1. Orbit and Constellation Calculations

The orbit calculation simulation code generates a time series of the satellite orbit in inertial-earth centered Cartesian and spherical coordinates. Each element in the time series represents a time step measured in seconds. Orbits are propagated for one day. Coverage results are repeatable from day to day because orbital periods are chosen to ensure a daily repeating ground track.

The constellation calculation simulation code calls the orbit calculation simulation code to propagate an entire constellation of orbits. The number of orbital planes is specified in the design vector. The orbital planes are priority allocated to regions of highest demand (i.e., the first satellite plane is used to serve the location with the highest user demand, the second satellite plane is used to serve the next highest demand location, etc.). Each orbital plane includes enough satellites to provide a continuous street of coverage within a $15^{\circ}$ band. A continuous street of coverage within the $15^{\circ}$ band helps to ensure that the constraint of servicing $90 \%$ of market demand at any point in time is met. The number of satellites within each plane is also evenly spaced apart in time in order to provide continuous access and coverage to the users. This number of satellites computation is done at perigee because a spacecraft provides the least amount of Earth coverage at perigee. If continuous coverage is achieved at perigee, it 
will guarantee coverage overlaps for the remainder of the satellite orbit with the greatest amount of overlap at apogee.

To validate the above functions, 2-D and 3-D orbit plotting routines were used to visually check that the correct orbits are calculated. Simulation results compared favorably to cases in which the orbit could be easily propagated analytically, e.g. geostationary spacecraft.

\section{Link Budget Calculation}

The link budget simulation module calculates the number of users that each satellite can accommodate. In this routine the number of channels per satellite is calculated based on the MF-TDMA and MF-CDMA capacity formulations developed and validated by de Weck and Chang (2003) to integrate the bandwidth and power limits on capacity. ${ }^{7}$ The link budget is calculated at every time step.

3. Spacecraft Module

The spacecraft module estimates spacecraft mass, volume, and other pertinent quantities using the scaling relationships developed and validated by de Weck, Springmann, and Chang (2003). ${ }^{10}$ For an elliptical satellite, the calculation is taken at apogee, providing the worst case scenario.

4. Launch Module

The launch module specifies the minimum cost launch option for the constellation from a set of currently available launch vehicles. When feasible, the module assigns multiple spacecraft per launch vehicle to reduce the number of launches required for system deployment. The module is adjustable to account for policy and availability constraints.

\section{Lifecycle Cost Calculation}

The lifecycle cost calculation module incorporates a variety of costs. First, launch costs for an elliptical satellite are determined by assessing the cost of launching the system into its perigee altitude and the cost of a kick motor for orbital maneuvering. The lifecycle cost of the elliptical satellite constellation is then calculated using cost estimating relationships (CERs) as described in Wertz and Larson (1999). ${ }^{1}$ This module also includes an estimated cost required for radiation hardening because some spacecraft may enter the Van Allen radiation belts. The fixed charge (about \$10B) associated with the LEO backbone constellation was computed using the parameter values listed in Table 2. These parameters were chosen based on the work conducted by de Weck and Chang (2002) using the Iridium system. ${ }^{6}$ The only remarkable exception is the satellite antenna power, which was increased from $400 \mathrm{~W}$ to $4000 \mathrm{~W}$ to offset the broadband service requirements.

Table 2: LEO Backbone Constellation Parameters.

\begin{tabular}{|l|l|c|c|}
\hline Symbol & Parameters & Value & Unit \\
\hline $\mathrm{C}$ & $\begin{array}{l}\text { Constellation type } \\
\text { (Polar or Walker) }\end{array}$ & Polar & {$[-]$} \\
\hline $\mathrm{H}$ & Orbital altitude & 780 & {$[\mathrm{~km}]$} \\
\hline Emin & Minimum elevation angle & 5 & {$[\mathrm{deg}]$} \\
\hline Pt & Satellite transmitter power & 4000 & {$[\mathrm{~W}]$} \\
\hline GT_dB & $\begin{array}{l}\text { Satellite antenna gain } \\
\text { (Parabolic antenna) }\end{array}$ & 24.3 & {$[\mathrm{dBi}]$} \\
\hline MA & $\begin{array}{l}\text { Multiple access } \\
(\text { MF-TDMA, MF-CDMA) }\end{array}$ & MF-TDMA & {$[-]$} \\
\hline ISL & $\begin{array}{l}\text { Intersatellite links } \\
(1=\text { yes, } 0=\text { no })\end{array}$ & 1 & {$[-]$} \\
\hline
\end{tabular}

\section{System-Level Validation}

The backbone LEO circular satellite system was validated and benchmarked by de Weck and Chang (2002) ${ }^{6}$ against various existing LEO satellite systems that have been launched, including Iridium and Globalstar. The backbone system performed well against the different benchmarks. Note that neither Iridium nor Globalstar provide the same types of services of the hybrid system under consideration; no deployed systems have attempted such widespread dissemination of internet data services. The increase in required data rates necessitates the purchase of more frequency spectrum and the employment of more sophisticated spectrum utilization techniques such MFTDMA and MF-CDMA. Additionally, due to the lack of elliptical constellation systems that have been launched into space, there was no valid system to benchmark the elliptical aspect of the hybrid constellation. Thus, in order to 
validate the elliptical constellation, checks were continuously made to ensure that the results made sense. By and large, the results from the elliptical constellation are satisfactory.

\section{Optimization Results}

\section{A. Pareto Optimality Results}

Table 3 summarizes the levels used for the full factorial evaluation of the tradespace. Figure 6 shows the optimization results and Pareto fronts. Figure 7 uses the same data as in Fig. 6 with the addition that each subfigure is color-coded by the value of the indicated design variables. Some trends are immediately recognizable from Fig. 7. For example, in subfigure (f), designs using MF-CDMA clearly outperform those using MF-TDMA regardless of the cost. The binary nature and clear distinction in performance allows the designer to easily see the advantage of MF-CDMA from subfigure (f). The same cannot be said for the other design variables. To better visualize trends in the other design variables, two novel visualizations of the trade space were developed for this optimization problem.

Table 3: Factor Levels for Full Factorial Evaluation of the Tradespace.

\begin{tabular}{|c|c|c|}
\hline Design Variable & Levels & Units \\
\hline $\mathrm{T}$ & $1,1 / 2,1 / 3,1 / 4,1 / 5$ & {$[$ days $]$} \\
\hline $\mathrm{E}$ & $0.001,0.1,0.3,0.5$ & {$[-]$} \\
\hline $\mathrm{NP}$ & $4,6,8,10$ & {$[-]$} \\
\hline $\mathrm{Pt}$ & $2,4,6,8$ & {$[\mathrm{~kW}]$} \\
\hline $\mathrm{DA}$ & $1.5,2,2.5,3$ & {$[\mathrm{~m}]$} \\
\hline $\mathrm{MAS}$ & MF-TDMA, MF-CDMA & {$[-]$} \\
\hline
\end{tabular}

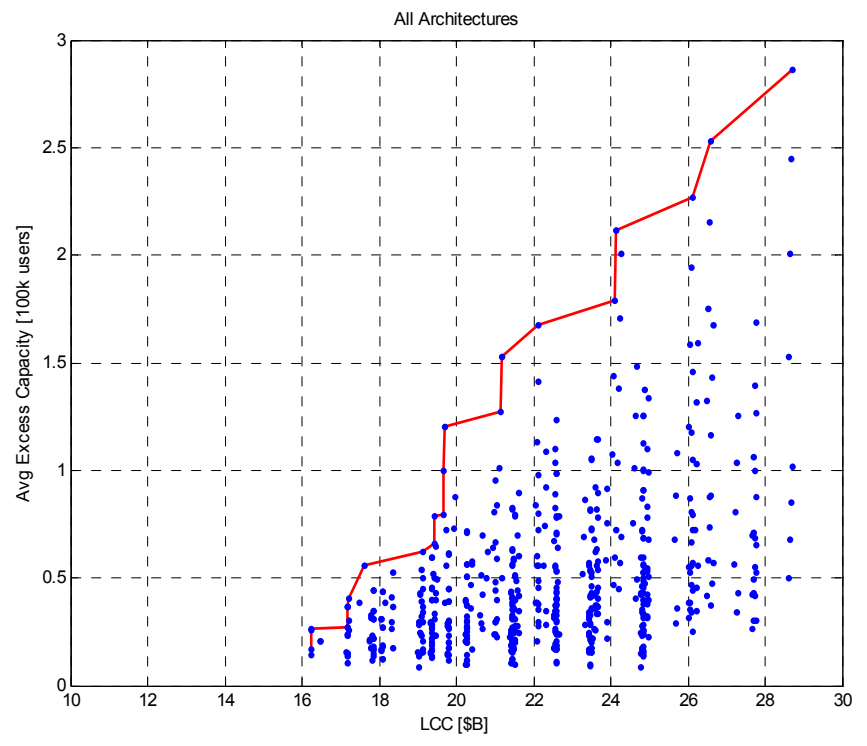

Figure 6. Pareto Front.

\footnotetext{
$\$$ To ensure a complete understanding of the results, the authors highly recommend viewing the results in color.
} 

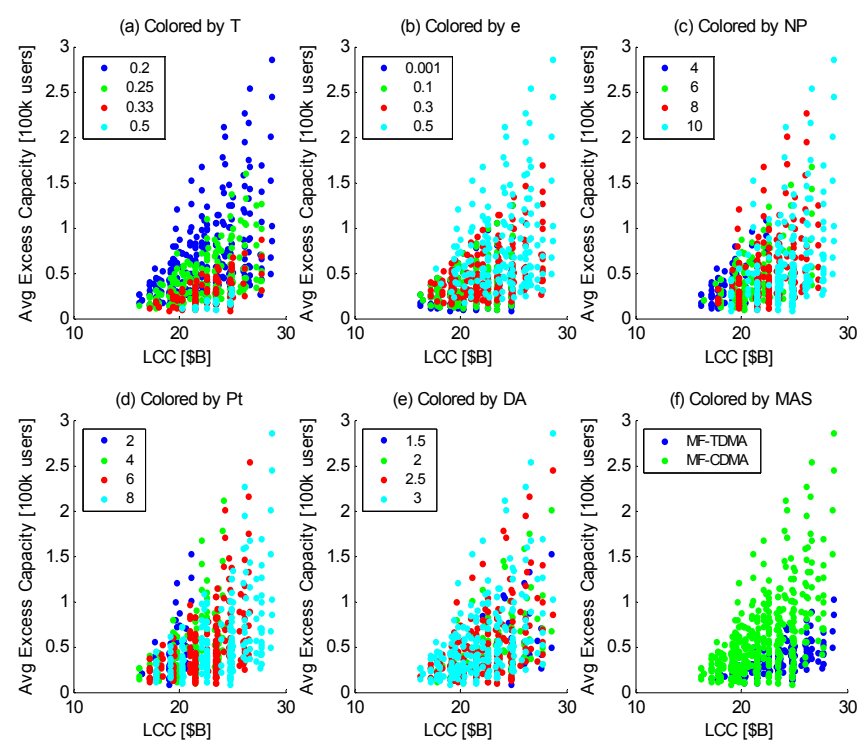

Figure 7. Trade Space Colored by Design Variable Choices.

The first technique is convex hulls. For each design choice, the convex hull of the colored points in Fig. 7 is drawn in Fig. 8. Each convex polygon identifies the smallest convex region in which all designs that include a particular design decision fall in the tradespace. The impact of each design choice on subsequent design freedom is made apparent to the designer. For example, if MF-TDMA is chosen, then the system will perform somewhere within the blue polygon in Fig. 8(f), regardless of the values of the other design variables ${ }^{\S}$. By intersecting corresponding polygons, the designer can visualize the impact of more complex design choices, e.g. a low power, high gain system vs. a high power, low gain system.
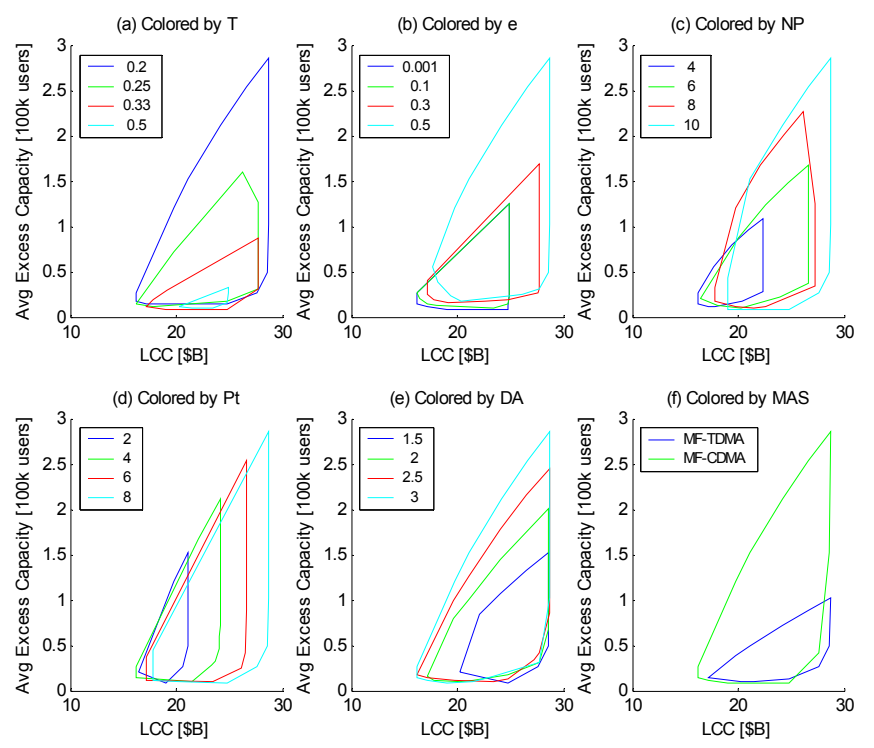

Figure 8. Convex Hull-based Visualization.

The second visualization technique is conditional Pareto fronts. Here, the Pareto front is plotted conditioned on holding one design variable fixed (see Fig. 9). With this visualization, dominance relationships between values of

$\S \S$ Technically, this conclusion is valid only for those values of the design variables enumerated. In practice though, if the enumeration is well representative of the actual design space, the conclusion will hold more generally. 
design variables become readily visible. For example, in Fig. 9(f) the conditional Pareto front for MF-CDMA is above and to the left of MF-TDMA. Because the objective is to minimize LCC and maximize AEC, MF-CDMA dominates MF-TDMA. Contrast this situation with Fig. 9(c) for LCC $<20 \$ B$ : designs with 4 planes dominate (just barely though). For LCC $>20 \$ B$, designs with 8 or 10 planes dominate those with fewer. Dominance relationships such as those described above can be used to make design choices while reducing the likelihood of trading away high value portion of the design space. The following example illustrates the technique.

From Fig. 9(a) and (f), it is clear that all members of the Pareto front have $\mathrm{T}=0.2$ days and MAS $=$ MF-CDMA. This is the first filter applied to the design space. After restricting the tradespace to only those designs with $\mathrm{T}=0.2$ days and MAS $=$ MF-CDMA, Fig 9 becomes Fig. 10. In Fig. 10, the dominance of DA $=3$ meters is apparent. The designer would then further restrict the tradespace to only those designs with DA $=3$. The new conditional Pareto fronts are plotted in Fig. 11. There is no longer clear dominance of the design variable values as was the case in Figures 7-10. Further design choices will be contingent upon the available budget. If the designer is certain that more that 20\$B is available, then an eccentricity of 0.5 can be said to dominate other choices of eccentricity. Restricting the tradespace further to those designs with $\mathrm{e}=0.5$ results in Fig. 12. A dominance relationship now exists for the NP design variable with 8 or 10 planes dominating. The design space is further restricted to designs with 8 or 10 planes (see Fig. 13). With the design decisions made thus far, the original design space of 2560 points (Fig. 7) has been reduced to eight designs (see Fig. 14). This is a small enough number that more detailed analysis can and should be carried out before eliminating any of the designs.
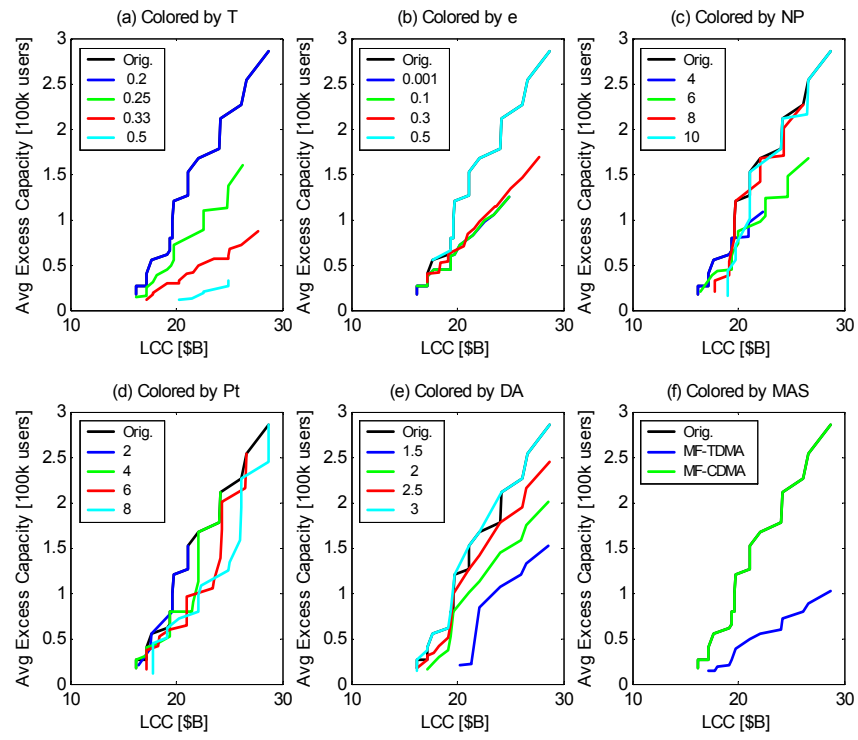

Figure 9: Pareto Fronts given each fixed Design Variable. 

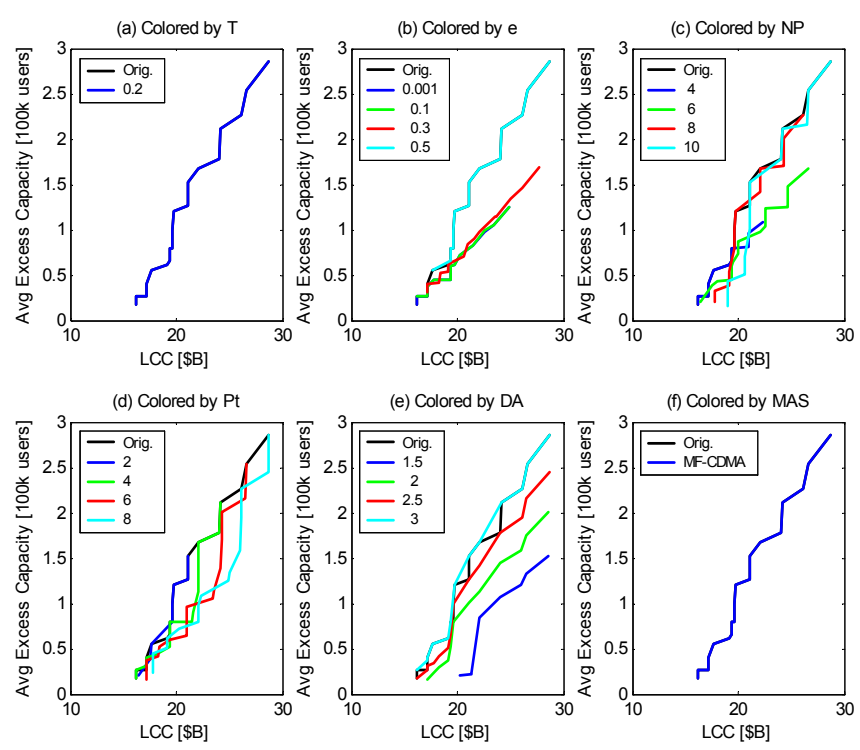

Figure 10: Conditional Pareto Fronts after fixing $T$ and MAS.
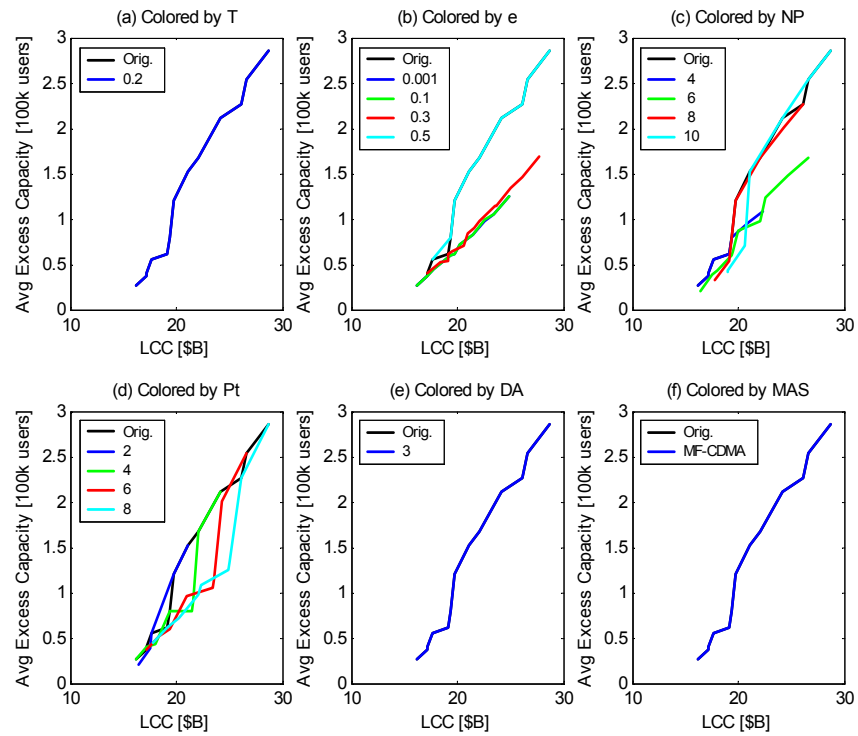

Figure 11: Conditional Pareto Fronts after fixing T, MAS and DA. 

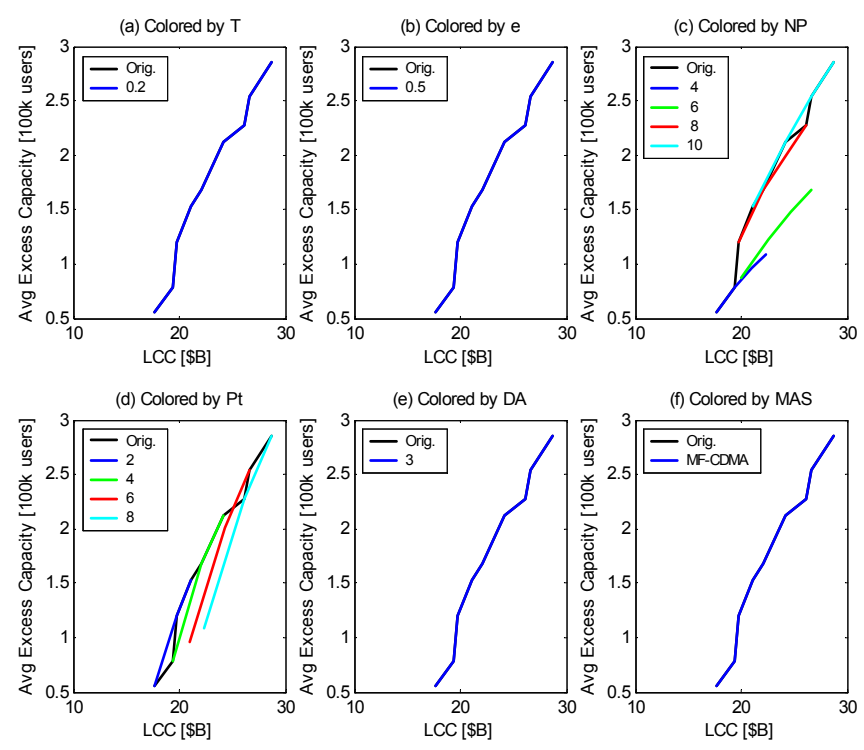

Figure 12: Conditional Pareto Fronts after fixing T, MAS, DA and e.
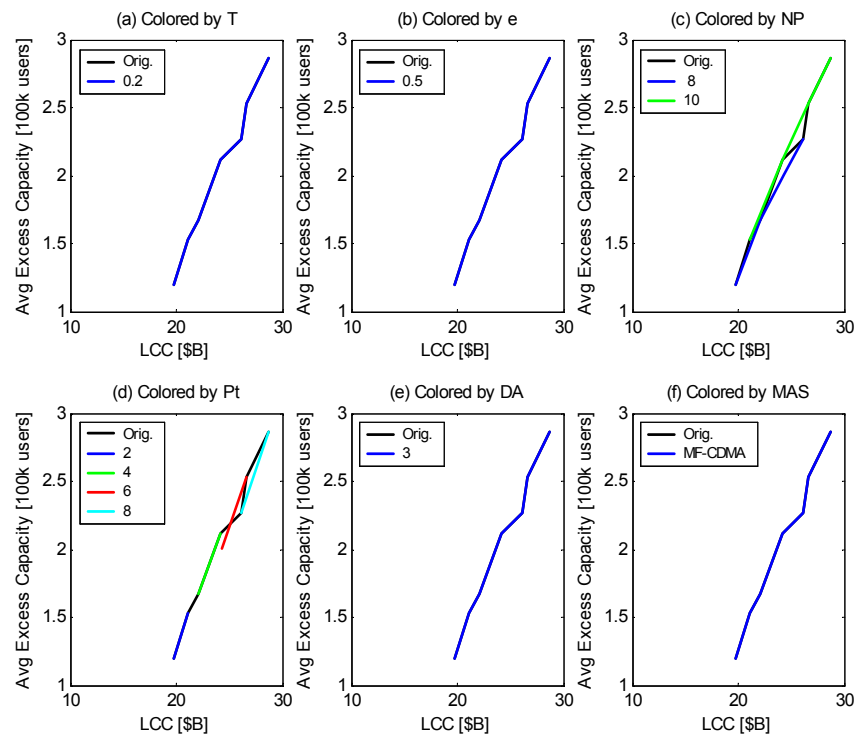

Figure 13: Conditional Pareto Fronts after fixing T, MAS, DA and e; NP restricted to 8 or 10. 


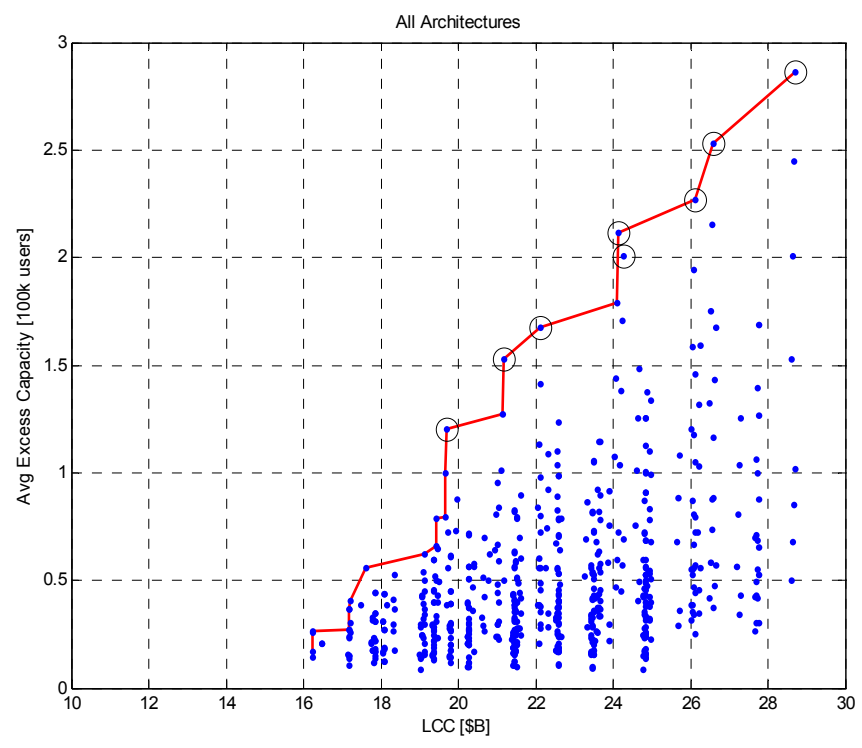

\section{Figure 14: Results of conditional Pareto Front analysis. Space of 2560 designs reduced to 8 (circled) candidates for further study.}

The power of the design methodology suggested in the example above is that at each step the designer makes choices based upon the entirety of his knowledge as captured in the tradespace. In practice, the model should be refined between every step by exploring the remaining space in greater depth (more levels per free design variable) and/or improving fidelity. Since each design choice reduces the area of the tradespace being explored, fidelity may be improved without suffering a significant penalty in computation time. With such a system, the precision of the designer's knowledge of the tradespace will increase to meet the later, more challenging, design decisions.

A brief discussion of parameter sensitivities follows. As the tradespace is highly discrete, the discussion will focus on the relative impacts of changes in parameter values on the design space. In the scenario discussed above, the earth transmitter gain was assumed to be $18 \mathrm{dBi}$. When this gain is increased to $37 \mathrm{dBi}$ (corresponding roughly to a $0.5 \mathrm{~m}$ parabolic antenna dish) and with all other parameters held fixed, the AEC more than doubles across all choices of the design variables. Likewise, reductions in the bandwidth occupied by the system seemed to have a drastic effect on the performance of the system. Changes in the percentage of satellite market capture and signal modulation level were found to noticeably impact the set of Pareto architectures when MF-TDMA was chosen; however, when MF-CDMA was chosen, little to no impact was observed. The tradespace appears to be most sensitive to changes in the earth transmitter gain and the amount of spectrum allocated to the satellite system by the FCC.

\section{Conclusions and Future Work}

It has been shown that hybrid constellations combining a global backbone with additional planes added to cover high demand areas can provide reasonable solutions to the broadband communication constellation design problem. The distribution of demand for broadband services is highly asymmetric, with a few peaks of high demand separated by large areas with low or no demand at all. This asymmetry of demand has led to failure modes existing in traditional design approaches (that do not account for this asymmetry). In one case, capacity, which in aggregate could serve the entire market, is uniformly distributed leading to excess capacity in low demand areas and a dearth of capacity in high demand areas, which are high revenue areas. This mismatch between demand and capacity makes such systems unprofitable and renders them under-utilized. In the other case, large satellites (or a large number of small satellites) are used to ensure demand is met all over the earth. Such systems will have extremely high start-up costs that prevent them from ever getting off of the ground.

Hybrid constellations solve this problem by first providing a baseline service (via the backbone) to start the market and then supplementing the backbone with strategically-placed spacecraft to serve high demand areas. Previous researchers have successfully used polar constellations to give higher fold coverage in high demand latitude bands. This paper extends that work by using elliptical orbits with repeating ground tracks to account for both longitudinal and latitudinal asymmetry in demand. The relative crudeness of the cost models used do not allow 
one to conclude that such a strategy is strictly better than the polar orbit-based strategies proposed by others, however hybrid designs do seem to show promise. As additional spacecraft are deployed in independent planes (i.e., one need not launch planes in pairs or triples), they can be deployed in a staged manner allowing the designer to react to observed demand instead of predicting future demand. Further research will be needed to verify and extend this conclusion. More generally, the results presented are important to the practice of constellation design.

Through conditional Pareto analysis, whereby the Pareto optimal set of designs given a particular design choice are identified, it was determined that Pareto optimal solutions to the hybrid constellation design problem will have a period of 1/5 day, eccentricity of 0.5 and will use MF-CDMA. These results hold irrespective of the other design choices. With MAS, eccentricity, and period fixed, an antenna diameter of 3 meters dominated other diameter length choices. Of the original 2560 designs enumerated only eight met all the criteria described in the results section. These eight should be considered for further, more detailed analysis. Power seems to be the key indicator of location along the Pareto Front for these eight designs. Therefore, improving the fidelity of the spacecraft module should also be a focus of subsequent design efforts. Viewing the conditional Pareto fronts and the convex hulls of each design choice allows one to come to these conclusions. The usefulness of these techniques even with the crude models used is reflective of their applicability to the problem of conceptual design.

The major open issues to be addressed in the development of this research include:

1. Optimizing the backbone constellation

Further work needs to be conducted on optimizing the backbone constellation within the hybrid satellite system model. The question of what level to set the threshold between the backbone and elliptic subconstellations is in itself an optimization problem that needs to be solved when minimizing LCC for the entire hybrid constellation.

2. Defining traffic patterns and traffic classes

Traffic patterns and traffic classes within this hybrid satellite system should be modeled. This will improve capacity calculations.

3. Coding for radiation shielding due to Van Allen Belts

Radiation shielding is necessary because a hybrid satellite system may have orbits that pass through or near the Van Allen Belts. The current cost estimation used for satellite hardening is taken as $2-5 \%$ of the total satellite cost. ${ }^{1}$ The simulation model presented currently uses this metric because a more sophisticated relation between cost and satellite hardening is not readily available.

\section{Obtaining accurate launch vehicle motor costs for the Geo-Transfer Orbit kick motors}

For the values being inputted, the launch cost module works correctly, thus once accurate values are obtained the module will be suitably improved. However, for the optimal cost to be reasonable, the actual costs and a table of available motors will need to be added to the simulation.

5. Modeling the hand-off problem

The handoff problem is not addressed in the current simulation. Satellite handoff modeling is a key component of interconnected network satellite simulations. However, it is difficult to encode and simulate handoffs. For example, a handoff management strategy (e.g., "make before break" or "break before make") must be implemented with considerations to bandwidth allocation, Quality of Service provisioning and mobility management. Maintaining connectivity between two asymmetrical satellite constellations is difficult. With more time, it is certainly something that should be considered for a more refined system model simulation analysis.

\section{Acknowledgments}

This work was completed for the Multidisciplinary System Design Optimization course at the Massachusetts Institute of Technology during the spring of 2003.

\section{References}

\footnotetext{
${ }^{1}$ Wertz, J. R. and Larson, W. J., Space Mission Analysis and Design, Microcosm Press \& Kluwer Academic Publishers, El Segundo, California \& Dordrecht, The Netherlands, 3rd ed., 1999.

${ }^{2}$ Rider, L., "Optimized Polar Orbit Constellations for Redundant Earth Coverage," The Journal of the Astronautical Sciences, Vol. 33, No. 2, April - June 1985, pp. 147 - 161.

${ }^{3}$ Adams, W. and Rider, L., "Circular Polar Constellations Providing Continuous Single or Multiple Coverage Above a Specified Latitude," The Journal of the Astronautical Sciences, Vol. 35, No. 2, April - June 1987, pp. 155 - 192.
} 
${ }^{4}$ Lang, T. J. and Adams, W. S., "A Comparison of Satellite Constellations for Continuous Global Coverage," Mission Design \& Implementation of Satellite Constellations, edited by J. C. van der Ha, International Astronautical Federation, The Netherlands, 1998, pp. $51-62$.

${ }^{5}$ Turner, A. E., "Constellation Design Using Walker Patterns," No. 2002-4636, August 2002, In AIAA/AAS Astrodynamics Specialist Conference and Exhibit Proceedings.

${ }^{6}$ de Weck, O. L., and Chang, D., "Architecture Trade Methodology for LEO Personal Communication Systems," AIAA Paper 2002-1866, May 2002, In 20th AIAA International Communication Satellite Systems Conference and Exhibit Proceedings.

${ }^{7}$ de Weck, O. L., and Chang, D., "Basic Capacity Calculation Methods and Benchmarking for MF-TDMA and MF-CDMA Communication Satellites," AIAA Paper 2003-2277, April 2003, In 21st AIAA International Communication Satellite Systems Conference and Exhibit Proceedings.

${ }^{8}$ Kashitani, T., "Development and Application of an Analysis Methodology for Satellite Broadband Network Architectures," AIAA Paper 2002-2019, May 2002, In 20th AIAA International Communication Satellite Systems Conference and Exhibit Proceedings.

${ }^{9}$ de Weck, O. L., and Willcox, K., "Lecture 4: Modeling and Simulation," MSDO Lectures, MIT, 2003.

${ }^{10}$ de Weck, O. L., Chang, D., Springmann, P. N., "A Parametric Communications Spacecraft Model for Conceptual Design Trade Studies," AIAA Paper 2003-2310, April 2003, In 21st AIAA International Communication Satellite Systems Conference and Exhibit Proceedings. 